\section{Compte rendu GRAT/TARMED}

Hans Heinrich Brunner

Mesdames, Messieurs,

Chères consœurs, chers confrères,

Vous trouverez sous www.tarmed.ch (CUG) une récapitulation des décisions de la Chambre médicale sur le GRAT et le TARMED, à l'exception de celles du 12 avril 2001.

Cette récapitulation et les commentaires concernant leur mise (ou non-mise) en application tiennent lieu de compte rendu du chef des négociations soussigné pour les années 1988 à 2001. Les explications se réfèrent uniquement aux propositions et aux décisions de la Chambre médicale; ces dernières sont contraignantes, contrairement aux décisions de la Conférence des présidents qui, pour des raisons statutaires, ont plutôt un caractère consultatif. Ce compte rendu est suivi d'une série d'explications de principe: 1. Le projet GRAT a été conçu pour la révision tarif médical AA/AM/AI et il a été financé par un crédit initial de Fr. 7000 000.-, puis par d'autres crédits. Le projet aurait pu se terminer en 1995 par la présentation du nouveau tarif médical AA/AM/AI (les objectifs initiaux étaient dès le départ irréalistes). Ces ressources étaient beaucoup trop restreintes, comme une comparaison avec des projets étrangers l'a montré; ainsi la création d'un tarif comparable aux Etats-Unis, le "cpt-4", a coûté plus de 150 millions de dollars. La conséquence fut que, ni d'une manière générale ni de la part de la FMH, il n'a été possible de créer une organisation de projet adéquate tant sur le plan du personnel que de l'infrastructure jusqu'en l'an 2000. Cela explique une partie des erreurs notoires commises dans certaines phases et aussi le fait que le soussigné n'a pas pu se retirer au niveau stratégique lors de son élection à la présidence.

2. Avec l'extension du projet GRAT/INFRA au domaine de la LAMal, sous le nom du TARMED, la situation est devenue tout à fait intenable. A cette époque - en 1996 - il aurait fallu créer une toute nouvelle organisation de projet avec plus de collaborateurs et de plus grands moyens financiers ainsi qu'une gestion plus stricte des opérations et des finances. Au lieu de cela, la direction GRAT a été complétée par une délégation du CAMS et de $\mathrm{H}+$; le GRATAL, et plus tard le TARMED, constituait la plaque tournante stratégique et opérationnelle qui, avec des moyens financiers quelque peu plus conséquents, devait supporter le projet. Le projet INFRA lancé en dernière minute dispo- sait de tellement peu de moyen en personnel et en infrastructure que l'objectif fixé, à savoir l'obtention de données sur les coûts hospitaliers, était dès le départ irréalisable. Il est même étonnant que l'on ait obtenu de tels résultats dans ces conditions.

3. Dès 1996, l'échéancier et l'ensemble de la planification du projet furent déterminés par le Département fédéral de l'intérieur (DFI) ou, plus précisément, par son exigence qu'on lui présente une structure tarifaire uniforme. A noter en passant, que la contribution du DFI à ce jour s'élève à exactement Fr. 0.00. Après coup, on peut quasiment qualifier de délétère l'entrée en matière des parties contractantes par rapport à cette exigence, d'autant plus que celle-ci ne repose pas sur la moindre base légale; la qualité et l'ajustage du projet en ont considérablement souffert. Une nouvelle comparaison avec l'étranger montre que des projets similaires se déroulent sur une période de 8 à 10 ans. Savamment orchestrée par un groupe bien connu de représentants des médias selon le slogan "Pourquoi n'est-ce pas encore prêt», la campagne des autorités politiques manque donc totalement de fondement objectif et il aurait fallu y répondre d'une autre manière que par une attitude complaisante. La collaboration de la délégation aux négociations dans cette entreprise peu digne s'explique peut-être par la forte pression exercée sur le corps médical dès le début des années 90 par le monde politique et la collectivité; on accusait les médecins de mettre des bâtons dans les roues de la santé publique, un reproche aussi stéréotypé qu'infondé.

4. Le projet GRAT était très fortement marqué par la volonté de rechercher le consensus; on ne voulait plus des tarifs de bazar ni d'ailleurs des négociations tournant à l'affrontement (une affirmation contre une autre affirmation). Cette attitude s'est avérée utile durant la première étape, la phase de construction, ... mais a masqué le fait que les assureurs et les médecins avaient des avis différents sur des questions fondamentales et qu'il ne pouvait en être autrement. Des personnes qui ont l'habitude de collaborer de manière constructive et développent par conséquent des relations cordiales ne peuvent pas, d'une minute à l'autre, mener sur le ton de la confrontation des négociations intransigeantes sur des questions de principe et on ne doit pas le leur demander. Rétrospectivement, il eût été plus judicieux de confier à d'autres personnes ces négociations fermes absolument indispensables, notamment sur le revenu de référence, le temps de travail normal, les taux d'exploitation, etc.

5. Cette attitude typiquement suisse de rechercher le consensus à presque n'importe quel prix explique aussi pourquoi certaines lacunes du projet clairement visibles depuis longtemps n'ont pas été 
abordées avec plus de détermination. Il s'agit notamment des lacunes du modèle et de la mécanique tarifaires auxquelles le projet de remaniement/reconception entend maintenant remédier. Sur ce point, les explications données afin qu'elles figurent au procès-verbal de la Chambre médicale étaient certainement insuffisantes; le soussigné aurait dû ici obtenir de meilleurs résultats en menaçant de rompre les négociations.

6. Depuis le milieu des années 90, il était visible que la rémunération des prestations chirurgicales et interventionnelles allait subir une baisse générale inopportune, même considérable pour certaines spécialités. On ne peut en aucun cas dire que la délégation aux négociations n'a pas reconnu ce fait et n'a pas procédé à une analyse correcte des mécanismes qui étaient à son origine. Le fait est que les tentatives pour obtenir la correction de ces points un à un par de longues négociations n'ont que partiellement porté leurs fruits, parce que toutes les autres parties à la convention rejetaient, dans le meilleur des cas, la demande, s'ils n'y étaient pas farouchement opposés. Il en a été ainsi surtout pour la représentation des hôpitaux qui, au cours de ces dernières années se sont contentés d'essayer d'obtenir la part de gâteau la plus grande possible pour la rémunération de l'infrastructure hospitalière. Là aussi, une rupture des négociations en 1998/99 aurait été la bonne solution, mais elle n'a pas été adoptée par peur que le DFI mette en vigueur son "propre tarif», une menace dont nous savons aujourd'hui qu'elle n'était que du vent.

7. On ne peut pas dire non plus que les sociétés de discipline médicale - la "base» - n'aient pas été intégrées dans les travaux. Par comparaison, aucun des projets apparentés connus n'a autant inclus la participation de ces groupes de médecins. Le dernier exemple marquant a été l'élaboration en Allemagne d'une structure tarifaire similaire $(\mathrm{EBM}=$ Einheitlicher Bewertungsmassstab), projet que les sociétés de spécialistes ont, dans le meilleur des cas, pu suivre du haut de la tribune. Il vaudrait en soi la peine de mener une étude afin de découvrir pourquoi le reproche de n'avoir pas été inclus dans les travaux revient avec une telle constance. Le résultat serait une réponse composite formée de fausses compréhensions du processus de développement, certainement d'erreurs de gestion de la délégation FMH dans certaines phases ou parties du projet, d'une attitude de retrait, voire d'opposition viscérale, de la part de certaines sociétés ainsi que du fait que la conscience historique de nombreux groupements s'étend rarement au-delà de la période administrative de ses dignitaires.

8. Au cours de ces 3 à 4 dernières années, en particulier depuis la prise en compte du domaine LAMal, les travaux ont de plus en plus - tout d'abord imperceptiblement - été marqués par des prescriptions sur le plan politique qui n'avaient rien avoir avec ces travaux. Souvenons-nous des innombrables exigences de respect de la neutralité des coûts qui, sans le moindre fondement légal, avaient et ont pour seul but d'introduire un budget global au sens d'une mise à mort librement consentie par le corps médical. On ne peut voir là qu'une provocation dans l'omission desdits politiciens de fixer les conditions-cadres nécessaires et de clarifier les questions en suspens. Celui qui considère ce travail comme un simple devoir scolaire et qui y met autant de zèle qu'un simple élève pour son cours de chant ne doit donc pas s'étonner du résultat. La question de savoir si des tables rondes tenues peu après minuit permettent de faire avancer la cause reste ouverte. Si lesdites tables rondes étaient à la base d'une autre manipulation politique pouvant mener à une machination électorale, alors on peut d'ores et déjà envisager un échec de l'entreprise TARMED dans le domaine LAMal.

9. La position du surveillant des prix est également un problème qui n'a pas été résolu. Ce dernier interviendra à nouveau au moment de l'introduction des tarifs cantonaux et ne se prononcera sûrement pas en faveur du corps médical. Cette intervention qui aura lieu à coup sûr et dont ni le contenu ni les effets ne peuvent être évalués, représente l'un des plus grands dangers pour le projet. Malgré le climat d'entente qui règne actuellement dans les négociations avec le CAMS, il n'est pas impossible qu'au terme desdites négociations, le surveillant des prix réduise à néant les résultats obtenus et que les assureurs qui, déjà par le passé ont soutenu activement la position du surveillant des prix lequel abondait par ailleurs dans leur sens, se soumettent en toute hypocrisie à son diktat.

10. Le soussigné est d'avis que la position de la FMH en matière de négociations s'est progressivement améliorée depuis 1998 où elle était à son niveau le plus bas. Si le projet, tel qu'il sera présenté à la Chambre médicale des 4 et 5 mai, devait ne pas être accepté, alors la situation se dégraderait à nouveau; et même d'importantes interventions politiques à long terme n'y changeraient rien. A ce propos, il convient de tenir compte du fait qu'aux yeux du public et des politiciens, la FMH représente un corps médical capable et coopératif. Si, en fin de compte, l'opinion selon laquelle les politiciens n'ont qu'à introduire les tarifs contre le gré du corps médical devait triompher à la Chambre médicale, la FMH perdrait non seulement toute possibilité d'action dans le domaine des tarifs médicaux, mais cela porterait un préjudice irréparable à la FMH en tant qu'organisation médicale indépendante. Cela ne doit pas signifier que la Chambre médicale, en cas d'acceptation $\mathrm{du}$ tarif $\mathrm{AA} / \mathrm{AM} / \mathrm{AI}$, puisse et doive renvoyer la convention-cadre LAMal en l'accompagnant d'exigences rigoureuses. 
11. C'est une illusion de croire que les négociations tarifaires se termineront avec la prise de décision finale sur le TARMED. Au contraire, elles resteront - en particulier les négociations sur les prix - au centre des débats de politique sanitaire au cours de ces prochaines années. Sur le plan tactique, il faudra donc garder à l'esprit la nécessité d'améliorer constamment la structure tarifaire et d'imposer les intérêts des médecins, par exemple par le biais du projet actuel de remaniement tarifaire. Il se peut que celui qui attend la grande confrontation finale à ce sujet assiste au crépuscule des dieux en blouse blanche, un rôle que, d'ailleurs, ils ne peuvent ni ne veulent jouer.

\section{Conclusion}

$\mathrm{Au}$ vu de ce qui précède et en prévision des négociations conventionnelles à venir, on peut tirer les conclusions suivantes:

- Le temps où les négociations étaient basées sur un système de milice et de démocratie de base est révolu. Au cours de ces deux dernières années, la FMH a mis sur pied un groupe de spécialistes tarifaires, qui travaillent de manière professionnelle, tant sur le plan des négociations que sur le plan technique. C'est également dans cet ordre d'idées que le réseau de référence et le système de saisie des données ont été élaborés et mis en œuvre. La FMH devient ainsi indépendante des intérêts (et, en clair, des chantages) de tiers.

- Le temps de la suprématie des négociations tarifaires cantonales est également révolu. Ce mode de négociation n'est plus adapté à une époque où même le système d'assurance sociale helvétique est soumis au marché européen et où les parties contractantes doivent absolument agir sur le plan national, et bientôt aussi sur le plan international. Ce constat n'est pas en contradiction avec la remarque suivante: la résistance du corps médical envers les diktats tarifaires devrait être organisée sur le plan local et régional.

- A l'avenir, les différents niveaux d'organisation (stratégie, négociations et opérations) ne devront plus être mélangés. Cela s'applique principalement aux acteurs du niveau des négociations qui n'auront plus accès aux autres niveaux.
- Les nouveaux objectifs ne peuvent être atteints qu'au moyen d'un projet dont le financement est assuré et qui est soumis à un contrôle strict, également au sein de la FMH, sur le plan des finances et du déroulement. Pour ce faire, il faut s'assurer que la poursuite de ces objectifs ne soit pas interrompue si les conditions précitées ne sont plus remplies ou si d'autres objectifs implicites ont été ajoutés. Le projet de remaniement/reconception sera le premier test de cette philosophie somme toute banale.

- Si l'on retire le projet aux médecins, par le biais de manipulations politiques masquées et implicites, alors ceux-ci devront se distancer clairement et immédiatement de l'exécution du projet. Toute disposition à coopérer dans de telles circonstances ne serait malencontreusement perçue que comme une faiblesse et une attitude de complaisance. Ce point doit être clair, en particulier en vue d'une intervention avant la lettre de la part du surveillant des prix: le contrat n'est rempli que si les résultats des négociations sont entièrement appliqués et si les éventuelles interventions du surveillant des prix sont acceptées d'un commun accord.

- Les négociations tarifaires doivent être comprises comme un travail de longue haleine. Le TARMED et le remaniement du TARMED ne sont que les étapes d'une marche sans fin qui mènera bientôt aux domaines suivants: forfaits/DRG, rentabilité/ rationnement, qualité des fournisseurs de prestations autorisés à établir des factures, etc. Les structures de la FMH, mais en particulier aussi ses processus de décision internes, doivent servir cet objectif. La FMH n'aura plus à surmonter de négociations tarifaires à la TARMED s'étendant sur plusieurs années, souvent menées à un niveau personnel et ne reculant devant aucune attaque; ne serait-ce que parce qu'il ne sera plus possible de trouver une personne normalement constituée disposée à endosser la responsabilité de tels travaux. Je souhaite à mes successeurs plus de collégialité qu'il ne m’a été donné d'en vivre. 